\title{
Cratering of a Solid Body Due to the Liquid Impact
}

\author{
Y.A. Semenov • G.X. Wu
}

Received: date / Accepted: date

\begin{abstract}
An impact between a high-speed liquid and a solid body leading to cratering during the impact is considered. The problem formulation is presented within the framework of a self-similar velocity potential flow for a liquid wedge impacting the solid wall. A linear constitutive relation is adopted which links the speed of cratering with the pressure and shear stresses. The solution procedure is based on the integral hodograph method. It converts the boundary-value problem for the complex potential in the fluid domain into a system of integral equations which are solved using the method of successive approximations. The obtained solution is used to investigate the cratering mechanics during the impact. The results are presented in terms of the interface shape, the free surface shape, streamline patterns, and pressure distribution along the interface.
\end{abstract}

Keywords Liquid/solid impact · Erodible body · Self-similar flows · Integral hodograph method.

\section{Introduction}

Liquid/structure impact is a widely observed natural phenomenon. While high speed liquid impacts can cause many adverse effects such as structural damage or failure, these processes are also widely used in many engineering fields.

Y.A. Semenov

Department of Mechanical Engineering, University College London, Torrington Place, London WC1E 7JE, UK

E-mail: semenov@a-teleport.com

G.X. Wu

Department of Mechanical Engineering, University College London, Torrington Place, London WC1E 7JE, UK

E-mail: g.wu@ucl.ac.uk for correspondence 
Jet-cutting and grinding, hydraulic coal mining, jet cleaning, rock drilling and surface penetrating through a shaped charge are some examples of applications. A common feature in these is the interface deformation, or body cratering during the liquid impact. This feature may also appear in raindrops on soil or granular materials, raindrops alighting onto an ice sheet which may be accompanied by processes of melting or solidification and cavitation erosion caused by high-speed jets generated at the final stage of vapour bubble collapse. A mathematical model to account for this impact phenomena associated with the deformation of the interface needs to be developed.

Mathematical modelling of liquid impact processes is a challenging problem due to rapid changes of the free-surface shape and liquid velocity in some local areas. One way to solve this kind of problem is based on applying the technique of matched asymptotic expansions (Howison, Ockendon \& Wilson [1], Howison, Ockendon \& Oliver [2,3], Korobkin [4], Oliver[5]). This is principally for the fully transient flow. In many cases, especially the initial stage and in some local areas during the impact, the flow may be treated as selfsimilar. Mathematically this means that the spatial and temporal variables can be combined into new ones and the problem no longer depends on time explicitly in the new system. There is a large body of work on the self-similar impact problems [6-9]. In these studies, however, the bodies are rigid whether their shapes are given or prescribed.

High speed jet/solid impacts accompanied by the penetration of liquid into a solid body or evacuation of the body material have received much attention in connection with shaped charge devices widely used in industrial applications. The jet created by the very rapid plasticization of a liner in a shaped charge device makes the impact pressure so high that it exceeds the yield stress of the solid material. It plasticizes the materials and craters the body. An advanced elastic-plastic free boundary model that takes into account the residual stresses produced by the moving plasticized region of the target has been presented by Novokshanov \& Ockendon [10]. They considered the target as a solid which was initially an unstressed and isotropic elastic material of infinite extent. The jet impact was modelled by a localized high pressure applied to the target. For shaped charge devices, the pressure in the jet may be so high that the plasticized region is much larger than the radius of the jet. The flows in both the jet and plasticized region of the target are mainly driven by pressure gradient rather than shear force and they behave like that of an ideal fluid. Therefore, it has been commonly accepted that such a problem can be modelled based on the theory for an ideal fluid, as in Birkhoff et al. (1948) [11].

Other examples associated with cratering are water-jet cutting of metals, soft and porous materials. In these cases the stress caused by the jet only slightly exceeds the yield stress of the target, and the thickness of the plasticized material is relatively small. Therefore, the shape of the crater affects the flow configuration and, consequently, the stress on the target. The problem is coupled through the fluid/structure interaction, and the rate of penetration will be affected by this coupling. Experimental investigation of this type of penetration was undertaken by Uth \& Deshpande [12]. They observed that 
deformation of the target interface caused backflow of the jet, which then increased the force exerted on the target and thereby the penetration rate.

For some porous materials, the rate of material evacuation caused by impact may also depend on the exposure time of the material to liquid. When the stagnation pressure of the jet is greater than the threshold pressure of the material, the material grains will become spalled. This means that when one layer of grains has been moved away the process starts from the beginning with a new layer [13].

In the present study we consider the case in which the solid material is eroded by the liquid impact. This may occur when the stress caused by the fluid pressure is beyond the yield stress of the material, or the interface during the impact may undergo melting or solidification. The plasticized or fragmented region of the solid is assumed to be much smaller than the crater region and the evacuated material is considered not to have a major effect on the flow. The latter assumption may not be valid in the region of high-speed tip jets evacuating the material however, from the previous studies of water impact flows [7], it has been found that the tip jets have only minor effects on the main flow. This has led to some theoretical approaches ignoring the tip jets, such as in the Wagner theory of water impacts.

The impacting flow interacts with the moving solid interface whose shape needs to be determined by the coupled fluid/cratering mechanics. The impacting liquid is in the form of a wedge. Its limiting case with zero inner angle and finite incoming flow rate becomes a rectangular jet. Specifically, we use a two dimensional self-similar velocity potential flow theory for the problem. The integral hodograph method $[8,14]$ is adopted. It enables the original boundaryvalue problem for the complex potential in the fluid domain to be converted into a system of integro-differential equations along the straight lines. The method has been successfully used in a variety of impact problems $[8,15,16]$. However, the present problem has some new challenges. Both the crater shape and the pressure on its surface are unknown. They have to be determined as a part of the solution from additional physical considerations governing the cratering. Corresponding changes in the integro-differential equations and solutions have to be made. In the following section the mathematical formulation of the problem and the solution procedure are first described. The numerical results are provided in Section 3 where various case studies corresponding to linear constitutive relation between the speed of cratering and the pressure and shear stresses are undertaken. Their analyses and physical implications are based on the streamline patterns, pressure distribution, free surface and interface shapes. 


\section{Formulation of the problem and the solution procedure}

2.1 Hydrodynamic model for the solid cratering.

There has been a substantial amount of work using various relationships for the stress and penetration rate. Here, we consider a case in which the following constitutive relation linking, linearly, the cratering velocity $V^{*}$ normal to the interface with both the normal pressure $P$ and the shear stresses $\tau$ is used:

$$
V^{*}=-R_{p} P-R_{\tau}|\tau|
$$

where $R_{p}$ and $R_{\tau}$ are the material-dependent constants. We assume that $\tau$ is mainly due to Reynolds shear stress and adopt the simplest turbulence model for its link with the velocity [17]:

$$
\tau=\lambda^{\prime} \rho V_{s}^{2},
$$

where $V_{s}$ is the tangential velocity of the liquid, $\lambda^{\prime}$ is an empirical factor and $\rho$ is the fluid density. By substituting Eq.(2) into Eq.(1) and dividing the result by $\rho V^{2}$, where $V$ is the speed of the incoming liquid, we can represent the constitutive relation in the non-dimensional form

$$
v^{*}=-r_{p} c_{p}-r_{\tau} v_{s}^{2}
$$

where $r_{p}=0.5 R_{p} \rho V, c_{p}=\left(P-P_{a}\right) /\left(0.5 \rho V^{2}\right)$ is the pressure coefficient, $r_{\tau}=R_{\tau} \lambda^{\prime} \rho V, v_{s}=V_{s} / V$ and $P_{a}$ is the atmospheric pressure. In order to determine the cratering velocity and the shape of the interface, the solution of the hydrodynamic problem needs to be found. The coefficients $r_{p}$ and $r_{\tau}$ are taken as zero if the stress caused by liquid impact is less than the yield stress or the threshold shear stress of the material, respectively. For the larger stress, the melting of the material may be assumed to mainly depend on fluid/cratering interaction, and the stress on the interface is near the yield stress of the material. The cratering caused by the shear stress may also depend on a smoothness of the interface, porosity and time of the exposure, etc. In these cases a more sophisticated model than that given by Eq. (3) should be applied. However, it will not affect the procedure of the solution presented below.

2.2 Formulation of the problem for liquid wedge impact on an erodible wall.

A symmetric liquid wedge of half-angle $\alpha$ is chosen as the incoming liquid to hit an erodible wall. The liquid is assumed to be inviscid and incompressible, the flow to be irrotational, and the incoming velocity of the liquid wedge to the body is constant. The gravity and surface tension effects are ignored as well as the wall material evacuated by the liquid. With these assumptions, the flow can be described by the velocity potential in the frame of reference attached to point $A$ on the moving interface. Point $A^{\prime}$ is the initial contact of the liquid wedge tip and the body surface. As there is no length scale, 
a)

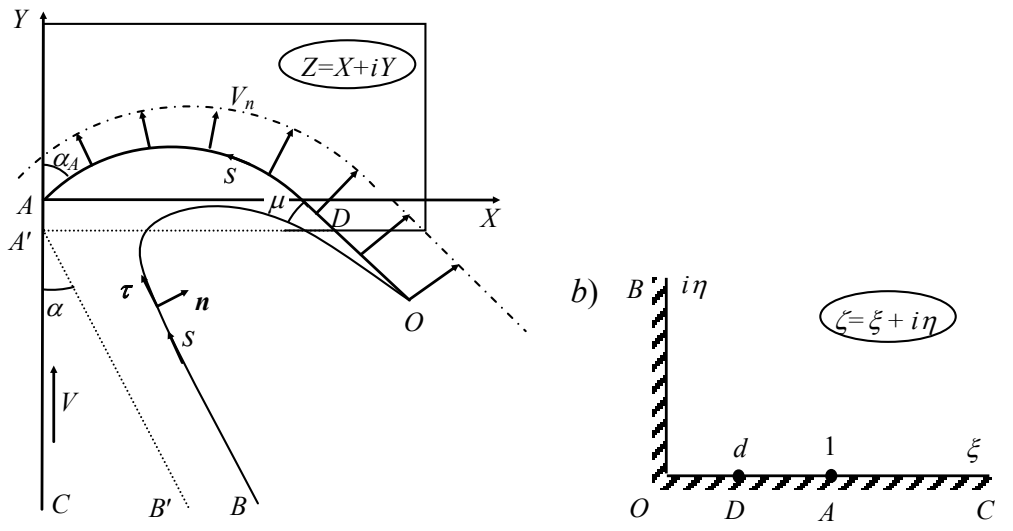

Fig. 1 Sketch of the cratering of the solid due to a liquid impact: the dotted line shows the liquid wedge at the time of impact; the dot-dashed line and arrows indicate the velocity profile of the cratering: $(a)$ physical plane and $(b) \zeta$-plane.

the interface deformation and the whole flow are self-similar. A sketch of the problem is shown in figure 1. The Cartesian coordinate system $X Y$ with origin at point $A$ is defined with $X$ axis in the direction of initially flat body surface and $Y$ axis along the symmetry line of the flow. The incoming liquid velocity $V$ relative to point $A$ is along the $Y$-axis, as shown in figure $1 a$. At point $D$ the flow detaches from the body and a splash jet with the tip angle $\mu$ may be formed. The fluid particle at point $A^{\prime}$ at $t=0$ will move to the jet tip $O$ after the impact. $A D$ is the interface which may form an angle $\alpha<\pi / 2$ with the $Y$-axis at point A.

For a constant impact velocity of the liquid wedge, the time-dependent problem in the physical complex plane $Z=X+i Y$ can be written in the stationary, or similarity plane $z=x+i y$ in terms of the self-similar variables $x=X /(V t), y=Y /(V t)$. The complex velocity potential $W(Z, t)$ can be written as

$$
W(Z, t)=V^{2} t w(z)=V^{2} t[\phi(x, y)+i \psi(x, y)]
$$

The problem is to determine the function $w(z)$. We choose the first quadrant of the $\zeta$-plane in figure $1 b$ as the region of the parameter variable $\zeta$ to derive expressions for the complex velocity, $d w / d z$, and for the derivative of the complex potential, $d w / d \zeta$, both as functions of the variable $\zeta$. Once these functions are found, the velocity field and the relation between the $\zeta$ - plane and the physical flow region can be determined as follows:

$$
v_{x}-i v_{y}=\frac{d w}{d z}(\zeta), \quad z(\zeta)=z_{A}+\int_{\zeta_{A}}^{\zeta} \frac{d w}{d \zeta^{\prime}} / \frac{d w}{d z} d \zeta^{\prime}
$$

where $z_{A}=0$ at the chosen origin of point $A$, and $\zeta_{A}=1$ is the image of $z_{A}$ in the $\zeta-$ plane as shown in figure $1 b$. 
The theorem about conformal mappings allows us to choose, arbitrarily, locations of three points in the $\zeta$ - plane, which are chosen as $O, B$ (a point at infinity) and $A$, as shown in figure $1 b$. In this plane, the positive part of the imaginary axis $(0<\eta<\infty, \xi=0)$ corresponds to the main free surface $O B$. The interval $0<\xi<d$ of the real axis corresponds to the free surface of the splash jet $O D$, and the interval $d<\xi<1$ corresponds to the wetted surface $A D$, and the rest of the positive real axis $d<\xi<\infty$ corresponds to the symmetry line $A C$. In the following formulation, the free surface $O D$ and the wetted surface $A D$ will be combined together and called interface. Their shapes will be determined together although the body surface boundary condition will be imposed on $A D$ and the constant pressure condition will be imposed on $O D$.

2.3 Derivation of the complex velocity and the function $d w / d \zeta$.

When the body surface is rigid, the normal component of the velocity equals zero, and therefore the velocity on the body surface is along its tangential direction. In the present problem the normal component of the velocity is nonzero due to cratering and can be written as

$$
v_{n}(s)=-v^{*}(s)-v_{A}^{*} \cos \left[\delta_{i}(s)\right], \quad s_{D}<s<0,
$$

where $v_{A}^{*}=v^{*}\left(s_{A}\right)$, and $\delta_{i}(s)$ is the slope of the interface, and $s$ is the arc length coordinate with $s=0$ at point $A$ and it decreases from point $A$ to point $D$. Therefore, the velocity direction on the body surface becomes unknown.

The boundary-value problem for the complex velocity function is formulated in the $\zeta-$ plane. At this stage we introduce function $\beta(\xi)=-\arg (d w / d z)$ along the interface $O A$, i.e. on the interval $0<\xi<1$ of the real axis of the $\zeta$ - plane, and function $v(\eta)$ defined along the positive part of the imaginary axis of $\zeta$ - plane, which is the speed of the liquid along the free surface $O B$. With these notations, we have

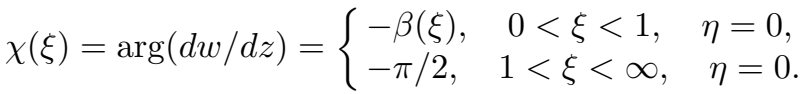

$$
\begin{aligned}
& v(\eta)=\left|\frac{d w}{d z}\right|, \quad 0<\eta<\infty, \quad \xi=0 .
\end{aligned}
$$

When we approach point $A$ along the interface $O A$, the velocity direction $\beta(\xi)=\tan ^{-1}\left(v_{y} / v_{x}\right)_{\xi \rightarrow 1-\varepsilon}, \varepsilon \rightarrow 0$, tends to the value $\pi / 2-\alpha_{A}$, or coincides with the direction of the interface. Along the $Y$-axis $\beta(\xi)=\tan ^{-1}\left(v_{y} / v_{x}\right)=$ $\pi / 2$. Therefore, there is a jump in the function $\beta(\xi)$ at point $\xi=1, \Delta \chi_{A}=$ $-\alpha_{A}$, when $\xi$ increases from $1-\epsilon$ to $1+\epsilon$.

As derived in $[8,14]$, the appropriate solution is given by:

$$
\frac{d w}{d z}=v_{\infty} \exp \left[\frac{1}{\pi} \int_{0}^{\infty} \frac{d \chi}{d \xi} \ln \left(\frac{\zeta+\xi}{\zeta-\xi}\right) d \xi-\frac{i}{\pi} \int_{0}^{\infty} \frac{d \ln v}{d \eta} \ln \left(\frac{\zeta-i \eta}{\zeta+i \eta}\right) d \eta+i \chi_{\infty}\right]
$$


where $v_{\infty}=v(\eta)_{\eta \rightarrow \infty}, \chi_{\infty}=\chi(\xi)_{\xi \rightarrow \infty}$. Using Eq.(7), Eq.(9) becomes

$$
\begin{aligned}
\frac{d w}{d z} & =v_{0}\left(\frac{1-\zeta}{1+\zeta}\right)^{\frac{\alpha_{A}}{\pi}} \\
& \times \exp \left[\int_{0}^{1} \frac{d \beta}{d \xi} \ln \left(\frac{\xi-\zeta}{\xi+\zeta}\right) d \xi-\frac{i}{\pi} \int_{0}^{\infty} \frac{d \ln v}{d \eta} \ln \left(\frac{i \eta-\zeta}{i \eta+\zeta}\right) d \eta-i \beta_{0}\right]
\end{aligned}
$$

where $v_{0}=v(0)$ and $\beta_{0}=\beta(0)$ are the speed and direction at point $O$. The functions $\beta(\xi)$ and $v(\eta)$ will be determined later from the kinematic and dynamic boundary conditions.

In order to obtain expression for the derivative of the complex potential, $d w / d \zeta$, it is useful to introduce the unit vectors $\mathbf{n}$ and $\boldsymbol{\tau}$ on the fluid boundary, which are normal and tangential to the surface, respectively. The former is directed outward from the liquid region, and while one moves in the $\boldsymbol{\tau}$ direction along the boundary, the arc length coordinate $s$ increases and the liquid region is on the left hand side (see figure $1 a$ ). With these notations, we have

$$
d w=\left(v_{s}+i v_{n}\right) d s,
$$

where $v_{s}$ and $v_{n}$ are the tangential and normal velocity components on the surface, respectively. Let $\theta(\eta)=\tan ^{-1}\left(v_{n} / v_{s}\right)$ and $\gamma(\xi)=\tan ^{-1}\left(v_{n} / v_{s}\right)$ denote the angles between the velocity vector with $\tau$ on the flow boundary. The function $\theta(\eta)$ is defined along the positive part of the imaginary axis of the $\zeta$-plane, which corresponds to the free surface $O B$ in the similarity plane, while $\gamma(\xi)$ is on the positive part of the real axis corresponding to the interface and the symmetry line. Eq.(11) allows us to determine the argument of the derivative of the complex potential, $\vartheta=\arg (d w / d \zeta)$, using the relation $d w / d \zeta=(d w / d s)(d s / d \zeta):$

$\vartheta(\zeta)=\arg \left(\frac{d w}{d \zeta}\right)=\arg \left(\frac{d w}{d s}\right)+\arg \left(\frac{d s}{d \zeta}\right)=\left\{\begin{array}{l}\gamma(\xi), \quad 0<\xi<1, \quad \eta=0, \\ \theta(\eta)+\pi / 2, \xi=0,0<\eta<\infty\end{array}\right.$

By analyzing the behaviour of the velocity angle along the whole flow boundary in figure $2 a$, we can see the variation of angle $\omega=\arg \left(v_{s}+i v_{n}\right)$. It is continuous along both the free surface $O B$ (defined as $\theta(\eta)$ ) and the interface $O A$ (defined as $\gamma(\xi)$ ), as shown by solid lines. $\theta(\eta)$ has step changes or discontinuities at points $O, B$ and $C$, while the function $\gamma(\xi)$ is continuous along the real axis. These step changes are shown by dashed lines in figure $2 a$.

Now we analyse the functions $\theta(\eta)$ and $\gamma(\xi)$ along the fluid boundary. Between points $C$ and $A, 1<\xi<\infty$, function $\gamma(\xi) \equiv-\pi$, since $v_{n}=0$ and $v_{s}<0$. When we move from point $A$ to point $O_{-}$along the interface, the function $\gamma(\xi)$ changes continuously from $\gamma_{A}=-\pi$ to $\gamma_{0}=\gamma(0)$ at point $O_{-}$. When we move in a counter-clockwise direction along an infinitesimal quarter of the circle centred at the point $\zeta=0$ in the parameter plane the corresponding line in the physical plane passes through the tip $O$ of the splash 


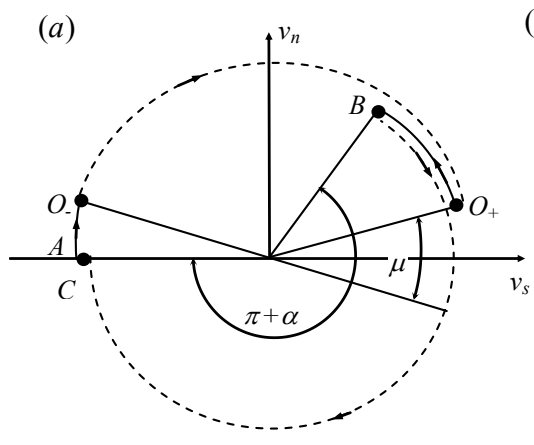

(b)

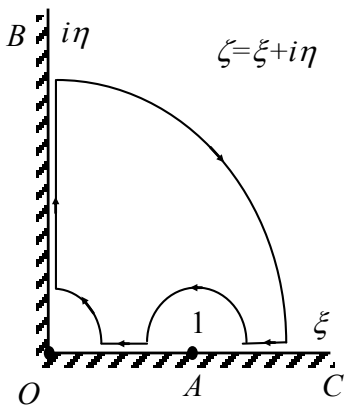

(c)

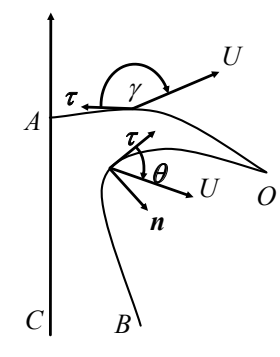

Fig. 2 (a) Variation of the velocity angle to the flow boundary, $\omega=\arg \left(v_{s}+i v_{n}\right)$ : the solid lines for continuous changes while the dashed lines for step changes. $(b)$ The corresponding variation in the parameter plane. (c) The definition of the angles $\theta$ and $\gamma, U$ is the velocity vector

jet. The jump in the function $\omega=\arg \left(v_{s}+i v_{n}\right)$ equals $\Delta=\mu-\pi$ as it can be seen from figure $2 a$, where $\mu=\theta_{0}+\pi-\gamma_{0}$, and $\theta_{0}=\theta(0)$. By taking into account Eq.(12) we can see that the jump in $\arg (d w / d \zeta)$ equals $\Delta_{\vartheta}=$ $\Delta+\pi / 2=\mu-\pi / 2$. The corresponding change of the $\operatorname{argument} \arg (\zeta)$ equals $\pi / 2$, and so we can expect that function $d w / d \zeta$ at point $O(\zeta=0)$ has a singularity of order $d w / d \zeta \zeta^{2 \Delta_{\vartheta} / \pi}$. When moving from point $O$ to point $B$ along the imaginary axis of $\zeta$-plane, the function $\theta(\eta)$ changes continuously from value $\theta_{0}$ to the value $\theta_{B}=\theta(\eta)_{\eta \rightarrow \infty}$.

We can write function $\vartheta(\zeta)$ as follows

$$
\vartheta(\zeta)=\arg \left(\frac{d w}{d \zeta}\right)= \begin{cases}-\pi, & 1<\xi<\infty, \quad \eta=0 \\ \gamma(\xi), & 0<\xi<1, \quad \eta=0 \\ \theta(\eta)+\Delta_{\vartheta}, & \xi=0, \quad 0<\eta<\infty\end{cases}
$$

The problem is then to find the function $d w / d \zeta$ in the first quadrant of the $\zeta$-plane which satisfies the boundary condition Eq. (13). This is a uniform boundary value problem, or $\arg (d w / d \zeta)$ is given on the entire boundary. As derived in $[8,14]$, the appropriate solution is given by:

$$
\frac{d w}{d \zeta}=K \exp \left[\frac{1}{\pi} \int_{\infty}^{0} \frac{d \vartheta}{d \xi} \ln \left(\zeta^{2}-\xi^{2}\right) d \xi+\frac{1}{\pi} \int_{0}^{\infty} \frac{d \vartheta}{d \eta} \ln \left(\zeta^{2}+\eta^{2}\right) d \eta+i \vartheta_{\infty}\right]
$$

where $K$ is a real factor, $\vartheta_{\infty}=\vartheta(\zeta)_{|\zeta| \rightarrow \infty}$. By performing the integration in Eq.(14) over steps where the value $\vartheta(\zeta)$ is known, we finally obtain the expression for the derivative of the complex potential in the $\zeta$-plane as

$$
\frac{d w}{d \zeta}=K \zeta^{2 \mu / \pi-1} \exp \left[\frac{1}{\pi} \int_{0}^{1} \frac{d \gamma}{d \xi} \ln \left(\xi^{2}-\zeta^{2}\right) d \xi+\frac{1}{\pi} \int_{0}^{\infty} \frac{d \theta}{d \eta} \ln \left(\zeta^{2}+\eta^{2}\right) d \eta\right] .
$$


Integration of Eq.(15) in the $\zeta$-plane allows us to obtain the function $w(\zeta)$ which conformally maps the first quadrant of the $\zeta$-plane onto the corresponding region in the complex potential plane:

$w(\zeta)=K \int_{1}^{\zeta} \zeta^{2 \mu / \pi-1} \exp \left[\frac{1}{\pi} \int_{0}^{1} \frac{d \gamma}{d \xi} \ln \left(\xi^{2}-\zeta^{2}\right) d \xi+\frac{1}{\pi} \int_{0}^{\infty} \frac{d \theta}{d \eta} \ln \left(\zeta^{2}+\eta^{2}\right) d \eta\right] d \zeta^{\prime}$,

where the complex potential at point $A(\zeta=1)$ is taken as zero without loss of generality.

Dividing Eq. (15) by Eq. (9), we can obtain the derivative of the mapping function

$$
\begin{aligned}
\frac{d z}{d \zeta} & =\frac{K}{v_{0}} \zeta^{2 \mu / \pi-1} \exp \left[\frac{1}{\pi} \int_{0}^{1} \frac{d \gamma}{d \xi} \ln \left(\xi^{2}-\zeta^{2}\right) d \xi+\frac{1}{\pi} \int_{0}^{\infty} \frac{d \theta}{d \eta} \ln \left(\eta^{2}+\zeta^{2}\right) d \eta\right. \\
& \left.-\frac{1}{\pi} \int_{0}^{1} \frac{d \beta}{d \xi} \ln \left(\frac{\xi-\zeta}{\xi+\zeta}\right) d \xi+\frac{i}{\pi} \int_{0}^{\infty} \frac{d \ln v}{d \eta} \ln \left(\frac{\eta-\zeta}{\eta+\zeta}\right) d \eta+i\left(\beta_{0}+\gamma_{0}\right)\right]
\end{aligned}
$$

The integration of this equation yields the mapping function $z=z(\zeta)$ relating the $\zeta$ - and similarity planes.

The pressure coefficient based on the ambient pressure, $P_{a}$, can be expressed through the pressure coefficient based on the pressure at the stagnation point $P_{A}$ as follows

$$
c_{p}=\frac{2\left(P-P_{a}\right)}{\rho V^{2}}=\frac{2\left(P-P_{A}\right)}{\rho V^{2}}-\frac{2\left(P_{a}-P_{A}\right)}{\rho V^{2}}=c_{p}^{*}-c_{p O}^{*},
$$

where

$$
c_{p}^{*}(\xi)=\Re\left(-2 w(\zeta)+2 z(\zeta) \frac{d w}{d z}(\zeta)\right)_{\zeta=\xi}-\left|\frac{d w}{d z}(\zeta)\right|_{\zeta=\xi}^{2}
$$

are obtained from Bernoulli's equation by choosing point $A$ as the reference point, where $w_{A}=0$, and taking the advantage of the self-similarity of the flow [16]. Here, we use that the pressure at point $O$ is equal to the ambient pressure.

The governing equations Eqs. (10) and (15) - (17) contain the unknown parameter $K$ and the functions $\gamma(\xi), \beta(\xi), \theta(\xi)$ and $v(\eta)$, all to be determined from physical considerations, as well as the dynamic and kinematic boundary conditions. The tip of the liquid wedge will depart from point $A$ to point $O$. We notice that when the flow is self-similar the velocity at point $O$ is constant. Thus, the position of point $O$ is determined by the velocity of the tip jet relative point $A, Z_{O}=V t z_{O}=V_{0} t e^{i \beta_{0}}=V t v_{0} e^{i \beta_{0}}$. The same argument was used Semenov, Wu \& Oliver [16] for the liquid/liquid impact. Thus, the 
distance between points $A$ and $O$ in the similarity plane equals $v_{0}$, which can be used to determine the parameter $K$ :

$$
K\left|\int_{0}^{1} \frac{1}{K} \frac{d z}{d \zeta}\right|_{\zeta=\xi} d \xi \mid=v_{0},
$$

where the integrand is determined from Eq. (17).

2.4 Determination of the functions $\theta(\eta)$ and $v(\eta)$ from boundary conditions on the free surface $O B$.

The dynamic and kinematic boundary conditions on the free surface $O B$ for an arbitrary self-similar flow can be obtained in the following form [8]

$$
\begin{gathered}
\frac{d \theta}{d s}=\frac{v+s \cos \theta}{s \sin \theta} \frac{d \ln v}{d s}, \\
\frac{1}{\tan \theta} \frac{d \ln v}{d s}=\frac{d}{d s}\left[\arg \left(\frac{d w}{d z}\right)\right] .
\end{gathered}
$$

Multiplying both sides of Eq.(21) and Eq.(22) by $d s / d \eta$ we obtain the following differential equation for the function $\theta(\eta)$ :

$$
\frac{d \theta}{d \eta}=\frac{v+s \cos \theta}{s \sin \theta} \frac{d \ln v}{d \eta},
$$

where $s=s(\eta)$ is obtained from integration of the expression $-|d z / d \zeta|_{\zeta=i \eta}$ along the imaginary axis of the parameter plane. Determining the argument of the complex velocity from Eq.(6) and substituting the result into Eq.(18), the following integral equation for the function $d(\ln v) / d \eta$ is obtained:

$$
-\frac{1}{\tan \theta} \frac{d \ln v}{d \eta}+\frac{1}{\pi} \int_{0}^{\infty} \frac{d \ln v}{d \eta^{\prime}} \frac{2 \eta^{\prime}}{\eta^{\prime 2}-\eta^{2}} d \eta^{\prime}=\frac{2 \alpha}{\pi} \frac{1}{1+\eta^{2}}+\frac{1}{\pi} \int_{0}^{\infty} \frac{d \beta}{d \xi} \frac{2 \xi}{\xi^{2}+\eta^{2}} d \xi
$$

2.5 Determination of functions $\gamma(\xi)$ and $\beta(\xi)$ from the kinematic boundary condition on the interface $O A$.

The normal component of the velocity on the interface, $v_{n}$, is determined by the constitute relation, Eq. (3), on part $A D$ of the interface. On $O D v_{n}$ is determined from the condition $P=P_{a}$ as $O D$ in reality is a free surface and is incorporated into the interface merely for the convenience of the mathematical formulation and procedure. Here, we may obtain an equation relating the velocity to the slope of the interface, given by the function $\delta_{i}(s)$, through exploiting the fact that the interface $Z_{i}=Z_{i}(S)=X_{i}(S)+i Y_{i}(S)$ in the physical plan is an expanding self-similar surface. By using the self-similar variable $z=Z /(V t)$ we can write $Z_{i}(S)=V t z_{i}(s)$, and the slope of the 
interface $\delta_{i}(S)=\delta_{i}(s)=\arg \left(d z_{i} / d s\right)$. With notation in Eq.(11) and using $d s /\left.d t\right|_{S}=-s / t$, we have

$$
d W=V^{2} t\left(v_{s}+i v_{n}\right) d s=\frac{d \bar{Z}_{i}}{d t} d Z=\frac{d \bar{Z}_{i}}{d t} V t \frac{d z_{i}}{d s} d s=V^{2} t\left(\bar{z}_{i}-e^{-i \delta_{i}} s\right) e^{i \delta_{i}} d s
$$

from which the normal component of the velocity of the interface is obtained as

$$
v_{n}(s)=\Im\left(\bar{z}_{i}(s) e^{i \delta_{i}(s)}\right),
$$

\section{where overbar denotes a complex conjugate.}

The tangential component of the velocity on the interface $O A$ can be determined from the real part of Eq. (11)

$$
v_{s}=\Re\left(\frac{d w}{d s}\right)=\Re\left(\frac{d w}{d z} \frac{d z}{d s}\right)=\Re\left(\left.\frac{d w}{d z}\right|_{\zeta=\xi} e^{i \delta_{i}[s(\xi)]}\right) .
$$

By using Eqs.(26), (27), and the definition of the function $\gamma(\xi)$ we can obtain

$$
\gamma(\xi)=\tan ^{-1}\left(\frac{\Im\left\{\bar{z}_{i}[s(\xi)] e^{i \delta_{i}[s(\xi)]}\right\}}{\Re\left\{d w /\left.d z\right|_{\zeta=\xi} e^{\left.i \delta_{i}[s(\xi)]\right\}}\right.}\right)
$$

Taking the argument of Eq.(17), the angle $\delta_{i}$ can be expressed $\delta_{i}=\beta+\gamma$. From this relation, we can obtain equation for the velocity direction

$$
\beta(\xi)=\delta_{i}[s(\xi)]-\gamma(\xi) .
$$

If $\delta_{i}(s)$ determining the shape of the interface $A O$ is a known function, then the Eq. (29) closes the system of integral equations Eqs. (23),(24),(28) for the unknown functions $\theta(\eta), v(\eta), \gamma(\xi)$ and $\beta(\xi)$.

\subsection{The shape of the interface.}

By equating the right hand side of Eqs. (6) and (26), we obtain the following equation for the function $\delta_{i}(s)$

$$
\Im\left(\bar{z}_{i}(s) e^{i \delta_{i}(s)}\right)=-v^{*}(s)-v_{A}^{*} \cos \left[\delta_{i}(s)\right] .
$$

The slope of the interface has angle $\delta_{i}\left(s_{A}\right)=\alpha_{A}-\pi$ at point $A$ (see figure $1 a$ ) which is unknown and it can be determined from the following physical considerations. A smooth concave shape of the interface occurs if the cratering velocity $v^{*}(s)$ decreases smoothly from point $A$ to point $O$, or $d v^{*} / d s \geq 0$. Then, due to the flow symmetry, it follows $\alpha_{A}=\pi / 2$. If the function $v^{*}(s)$ increases from point $A$, or $d v^{*} / d s \leq 0$, the shape of the interface becomes convex. A convex interface expanding in self-similar way is not practical, as it would lead to the self-crossing of $Z_{i}(S, t)$ in the physical plane $Z$. However, for $0<\alpha_{A}<\pi / 2$ a concave shape of the interface may exist. Point $D$ moves with 
the speed $v_{D}=v^{*}\left(s_{D}\right) / \sin \left[\delta_{i}\left(s_{D}\right)\right]$ which is the velocity of lateral expansion. Therefore, the angle $\alpha_{A}<\pi / 2$ should satisfy the following condition

$$
x_{D} \sin \delta_{D}=v^{*}\left(s_{D}\right),
$$

where $x_{D}=X_{D} /(V t)$ is the $x$-coordinate of the point $D$ in the similarity plane.

2.7 Determination of the shape of the free surface $O D$.

On the free surface $O D$, which is treated as the part of the interface in the present formulation, the pressure is equal to the ambient pressure. This is the condition enabling us to determine $\delta_{i}(s)$ on the interval $s_{O}<s<s_{D}$, corresponding to the interval $0<\xi<d$ on the real axis of the $\zeta$ - plane (see figure $1 b)$. First, we determine the new speed by using Bernoulli's equation in Eq. (19), taking into account that the pressure coefficient equal to $c_{p O}^{*}=c_{p}^{*}(0)$ on this part of the interface. Thus, from Eq. (19), we have,

$$
v_{i}(\xi)=\left|\frac{d w}{d z}\right|=\sqrt{2 \Re\left(-w(\zeta)+z(\zeta) \frac{d w}{d z}(\zeta)\right)_{\zeta=\xi}-c_{p O}^{*},} \quad 0<\xi<d .
$$

Here, the parameter $d$ is determined from $d=s^{-1}\left(s_{D}\right)$, the inverse function of $s=s(\xi)$. At the second step we seek the new velocity direction along $O D$. By taking the magnitude of Eq.(10) and equating it to $v_{i}(\xi)$, we obtain the following integral equation for the new approximation of the function $d \beta / d \xi$,

$$
\begin{aligned}
\int_{0}^{d} \frac{d \beta}{d \xi^{\prime}} \ln \left|\frac{\xi^{\prime}-\xi}{\xi^{\prime}+\xi}\right| d \xi^{\prime} & =\ln \left[\pi \frac{v_{i}(\xi)}{v_{0}}\left(\frac{1+\xi}{1-\xi}\right)^{\alpha_{A} / \pi}\right] \\
& -\int_{0}^{\infty} \frac{d \ln v}{d \eta} \tan ^{-1}\left(\frac{\xi}{\eta}\right) d \eta-\int_{d}^{1} \frac{d \beta}{d \xi^{\prime}} \ln \left|\frac{\xi^{\prime}-\xi}{\xi^{\prime}+\xi}\right| d \xi^{\prime}
\end{aligned}
$$

From the solution of this equation, we obtain

$$
\beta(\xi)=\beta_{D}+\int_{d}^{\xi} \frac{d \beta}{d \xi^{\prime}} d \xi^{\prime}, \quad 0<\xi<d .
$$

where $\beta_{D}=\beta(d)$ is known from the solution on the body surface $A D$ in the previous subsection. Then, the slope of the free surface $O D$, corresponding to the interval $0<\xi<d$ is obtained using the relation $\delta_{i}(\xi)=\beta_{i}(\xi)+\gamma_{i}(\xi)$, where the function $\gamma(\xi)$ is determined by Eq.(29).

The numerical solution of the system of equations, which is strongly nonlinear, is based on the procedure of successive approximations. The relaxation coefficients used in the iterations are chosen as $1 / 5$ for the functions $\beta(\xi)$ and $\gamma(\xi)$ and as from 0.001 to 0.1 for the function $\theta(\eta)$, depending on the value of 
angle $\mu$. It starts with $\beta(\xi) \equiv \beta_{0}, \gamma(\xi) \equiv-\pi, \delta_{i}(s) \equiv-\pi+\beta_{0}, v_{n}(\xi) \equiv 0$ on interface $O A$ and $\theta \equiv \alpha, v(\eta) \equiv 1$ on free surface $O B$, as initial values.

From Eq. (20), $K$ is obtained with using Eqs.(10), (15) and (16), respectively to obtain $d w / d \zeta$ and $w(\zeta)$, while $\operatorname{Eq}(17)$ is used to obtain $d z / d \zeta$. New approximation of the functions $\gamma(\xi)$ and $\beta(\xi)$ are then determined from Eq.(28) and Eq.(29), respectively, and the shape of the interface $\delta_{i}(s)$ is determined from Eq.(30). The functions $\theta(\eta)$ and $v(\eta)$ are determined from Eq. (23) and Eq.(24), respectively, which give the free surface shape $O B$. The iteration returns to Eq.(20) and the process is repeated until the convergence has been achieved.

\section{Numerical results}

\subsection{Numerical approach.}

The numerical approach employed in the present study is based on the method of successive approximations, which is similar to that used by Semenov \& Wu [15], for solving self-similar impacts between an impermeable solid body and a liquid wedge. Let us consider two sets of points distributed along the part of the real axis, $0<\xi_{j}<1, j=1 \ldots M$, and the imaginary axis, $0<\eta_{j}<\eta_{N}$, $j=1 \ldots N$, where $\eta_{N}$ is sufficiently large. The integrals within each segment in the system of equations are evaluated explicitly, using the linear interpolation for the functions $\gamma(\xi), \beta(\xi), \theta(\eta)$ and $v(\eta)$, on the intervals $\left(\xi_{j-1}, \xi_{j}\right)$ and $\left(\eta_{j-1}, \eta_{j}\right)$, respectively. The results contain the unknowns coefficients $\Delta \gamma_{j}=$ $\gamma_{j}-\gamma_{j-1}, \Delta \beta_{j}=\beta_{j}-\beta_{j-1}, \Delta \theta_{j}=\theta_{i}-\theta_{j-1}$ and $\ln \left(v_{j} / v_{j-1}\right)$, which are determined from the system of equations Eqs. (23), (24), (28), (29).

3.2 Impact between a liquid wedge and a cavity expanding into a solid wall.

We first consider a case in which the shape of the interface evolves in a prescribed way in the form of an expanding circular cavity. If the radius $R$ of the circle expands in the manner of $R=V^{*} t$, its slope as a function of spatial coordinate $s$ can be given as

$$
\delta(s)=-\pi+s / r, \quad s_{D}<s<O .
$$

where $r=V^{*} / V=v^{*}$ is the dimensionless radius of the cavity in the similarity plane, and $s$ is the arc length coordinate along the interface $A D O$, from $s=0$ at point $A$ to the $s_{D}=-\pi r / 2$ at point $D$ if the fluid departs from the cavity. When the arc length $A D,-s_{D}<\pi r / 2, O$ and $D$ will merge and will be inside the cavity. Otherwise the $O D$ part of the interface in figure $1 a$ is outside the cavity, and is part of the free surface in reality.

In figure 3 are shown the streamline patterns, free surfaces (solid lines), and the pressure distribution for the solid wall with cavity expanding with different velocities. In figure $3 a$ with $v^{*}=1.023$, the arc length $-s_{D}<\pi r / 2$ 


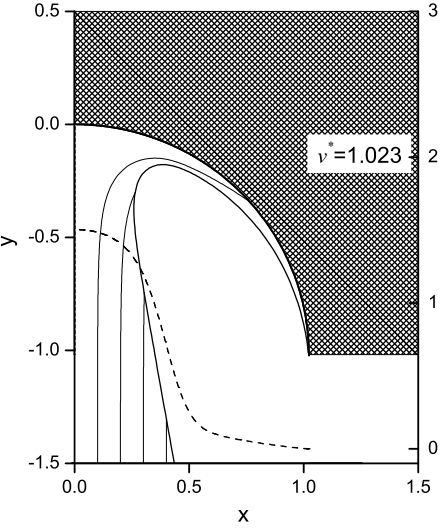

$(a)$

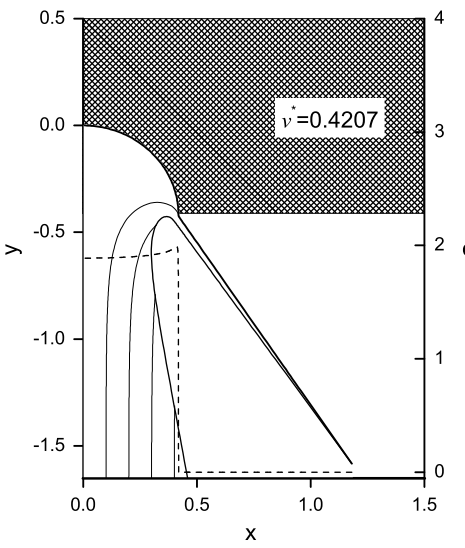

$(c)$

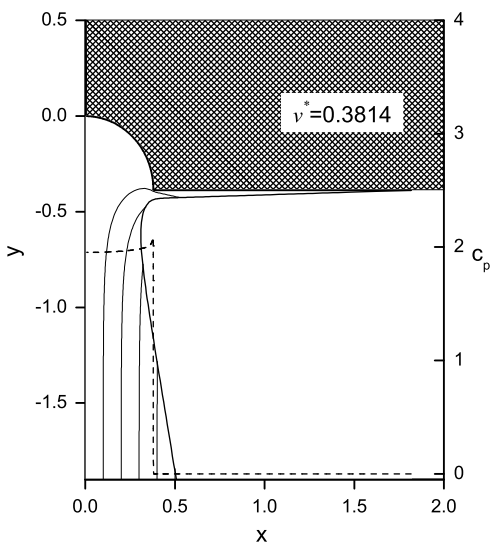

$(e)$

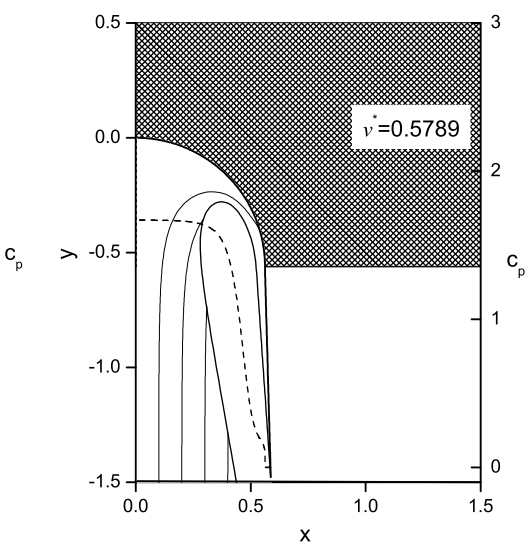

(b)

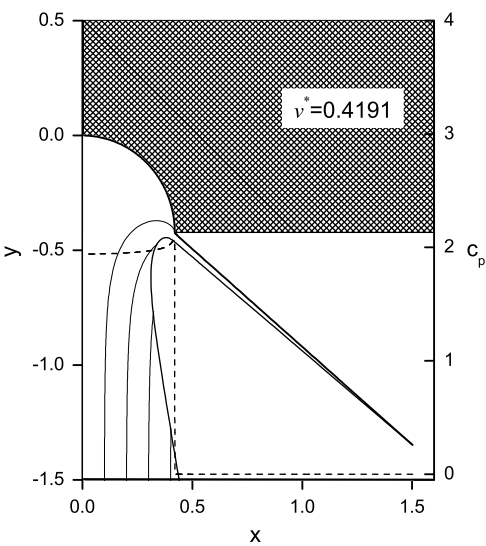

$(d)$

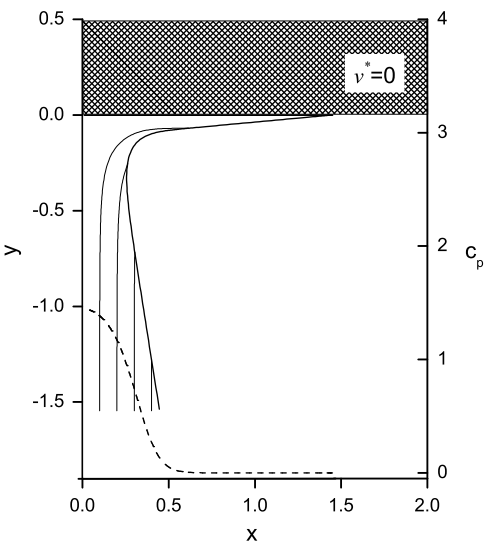

$(f)$

Fig. 3 Streamline patterns, free surfaces (solid lines), and the pressure distribution (dashed lines) for water impact between a liquid wedge of half-angle $\alpha=10^{\circ}$ and the solid wall with circular cavity expanding with radial velocity $(a) v^{*}=1.023,(b) v^{*}=0.5789,(c)$ $v^{*}=0.4207,(d) v^{*}=0.4191,(e) v^{*}=0.3814$ and $(h) v^{*}=0$. 
and there is no flow detachment. The maximal pressure coefficient is about $c_{p} \approx 1.5$ and it is located at the stagnation point, or the relative velocity to the fluid particle at the point is zero, in the cavity. For the smaller velocity of expansion, $v^{*}=0.5789$, shown in figure $3 b$, the liquid departs from the cavity surface and moves in the direction almost opposite to the incoming flow, and a splash jet is formed. Near the point of detachment on the cavity, the liquid layer is also very thin and it is virtually the extension of the splash jet. The pressure in this part of the cavity drops to zero rapidly. Due to the motion inertial, the splash jet keeps its direction of the slope of the cavity, from which it has departed. The pressure coefficient on the rest of the cavity, which takes about one third of the body surface, remains almost constant $c_{p} \approx c_{p} \max \approx 1.67$. For the velocity of the cavity expansion $v^{*}=0.4207$ in figure $3 c$ the splash jet does not extend into the cavity surface. The pressure inside has very small variation but it drops very fast from its maximal value $c_{p} \max \approx 2.0$ to zero near the point of detachment. The splash jet deviates from the vertical direction towards the main wall surface. A small reduction of the velocity of expansion from $v^{*}=0.4207$ in figure $8 c$ to $v^{*}=0.4191$ in figure $3 d$ substantially increases the deviation of the splash jet while the pressure changes very little. When the location of the pressure peak approaches ever closer to the cavity edge and as the jet no longer extends into the cavity surface, it is expected there is higher pressure zone inside the liquid near the cavity edge, which pushes the splash jet to bend towards the wall. Further decrease of the cavity to the value $v^{*}=0.3814$ leads to the intersection of the splash jet and the wall. In this case, the liquid is assumed to be fully attached to the body and the free surface boundary condition on the upper surface of the jet is replaced by the kinematic condition of the body surface. The maximal pressure coefficient remains about the same, $c_{p} \max \approx 2.0$ as it is seen from figure $3 e$. In the limiting case $v^{*}=0$ shown in figure $3 f$, cavity disappears and it corresponds to the impact of the liquid wedge and the non-erodible rigid wall.

\subsection{Impact by a liquid wedge on an erodible solid wall}

In reality, the cratering is quite a complicated physical process involving normal and tangential stresses as well as the material properties. In the idealized case in Eq.(1) only the hydrodynamic pressure and the shear stress are included in the constitutive relation in a particular manner. In order to study their effects separately, we will investigate two limiting cases of Eq.(3). The first corresponds to $r_{\tau}=0, r_{p} \neq 0$ and the second to $r_{\tau} \neq 0, r_{p}=0$.

For the first case we may note that $c_{p}=0$ at point $D$, and Eq.(3) therefore gives $v^{*}=0$, or the cratering velocity is also zero at the edge of the crater. In this case the crater edge should remain at the origin, that results in a closed cavity. Such a mathematical solution does not reflect the physics. Therefore, we introduce a minimal velocity $v_{\min }^{*}$ for the present case $r_{\tau}=0$. 
Then, the cratering velocity can be represented in the form

$$
v^{*}(s)=\max \left[v_{A}^{*} \frac{c_{p}(s)}{c_{p A}}, v_{\text {min }}^{*}\right] .
$$

where $v_{A}^{*}=r_{p} c_{p A}$. The interface and other flow parameters for impact between a liquid wedge of $\alpha=10^{\circ}$ and an cratering wall, based on Eq.(36) are shown in figure 4 at different ratios of $v_{\min }^{*} / v_{A}^{*}$. In all these cases the pressure is nearly constant in a region near the impact centre, which corresponds to nearly constant cratering velocity or a nearly circular shape of the body surface. The pressure drops rapidly near the crater edge, where the condition $v^{*}(s) \geq v_{\min }^{*}$ is applied. When a smaller $v_{\min }^{*}$ is chosen, the $x$ coordinate of $D$ will be obviously smaller and crater will have a smaller opening. As the fluid departs from the solid surface at point $D$ along its tangential direction, the formed jet will bend towards the incoming liquid wedge, as can be seen in figures $4 b, c$, and the jet may overlap with the main flow to cause a secondary impact. The overlapping is ignored in the present study. More detailed discussions and the reasons can be found in Semenov, Wu \& Oliver [16].

We now consider the case with $r_{\tau} \neq 0$ and $r_{p}=0$ in Eq.(3). From Eqs.(2) and (3) it follows that both shear stress and the speed of cratering at the stagnation point $A$ are equal to zero, since $v_{s}(0)=0$ due to symmetry. Therefore, the position of point $A$ remains at the original place $A^{\prime}$ during the impact process. As the interface expands with the normal velocity, which is expected to be positive, its shape near point $A$ is concave.

In figure 5 are shown the results for impact of a liquid wedge of $\alpha=30^{\circ}$ and the wall at different coefficient $r_{\tau}$. For a relatively small value of the coefficient $r_{\tau}$ shown in figure $5 a$ only small amount of the material is removed. However, even for small value of $r_{\tau}$ some hollow appears which causes the detachment of the liquid from the body surface and formation of the splash jet. For the case of $r_{\tau}=0.5$ shown in figure $5 b$ the size of the crater in the $x$-direction becomes smaller, while the crater depth, the length of the splash jet and the angle between the wall and the splash jet become larger. The corner at the point $A$ becomes sharper as the coefficient $r_{\tau}$ increases. This affects the pressure coefficient at the origin, which becomes smaller. The length and angle of the detached splash jet to the wall in figures $5(b-d)$ is only slightly increased. For all the cases in figure 5 near the root of the splash jet the pressure coefficient drops rapidly. Therefore, there is no force which pushes the splash jet bending towards the wall, as it is in figure $3 e$.

\section{Concluding remarks}

We have developed a method which generalizes previous studies on liquid/solid impact and accounts for certain essential features associated with cratering of a solid body. The focus is on those configurations where the flow can be treated as self-similar, which can be realistic at earlier stages of the impact. The mathematical approach is based on the integral hodograph method, which 


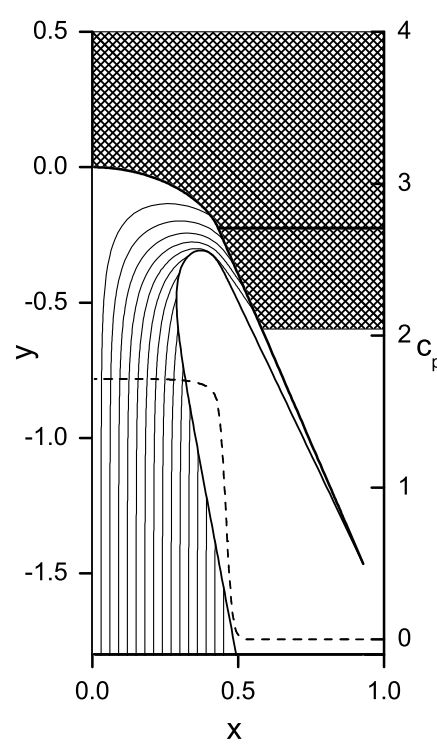

(a)

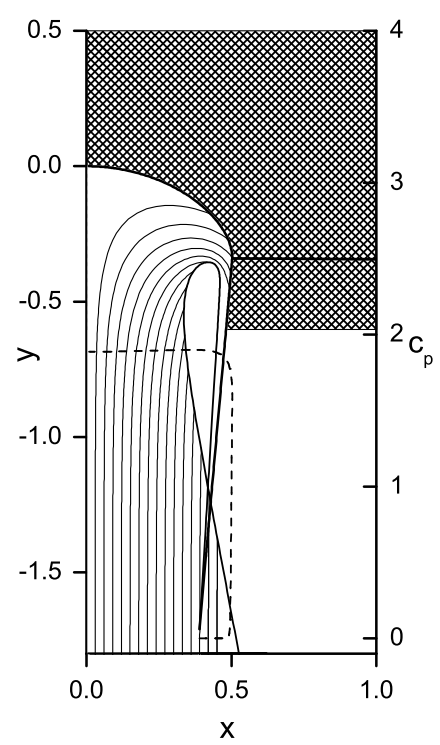

(b)

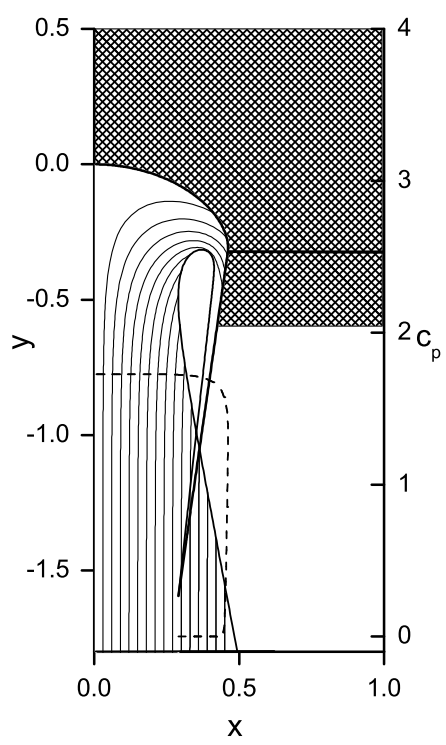

(c)

Fig. 4 Impact between a liquid wedge of half-angle $\alpha=10^{\circ}$ and an initially flat solid wall based on Eq.(36) with $v_{A}^{*}=0.6(a) v_{\min }^{*} / v_{A}^{*}=0.9,(b) v_{\min }^{*} / v_{A}^{*}=0.8,(c) v_{\min }^{*} / v_{A}^{*}=0.7$. The interface between the wall side and thick line correspond to $v^{*}(s)=v_{\min }^{*}$. 

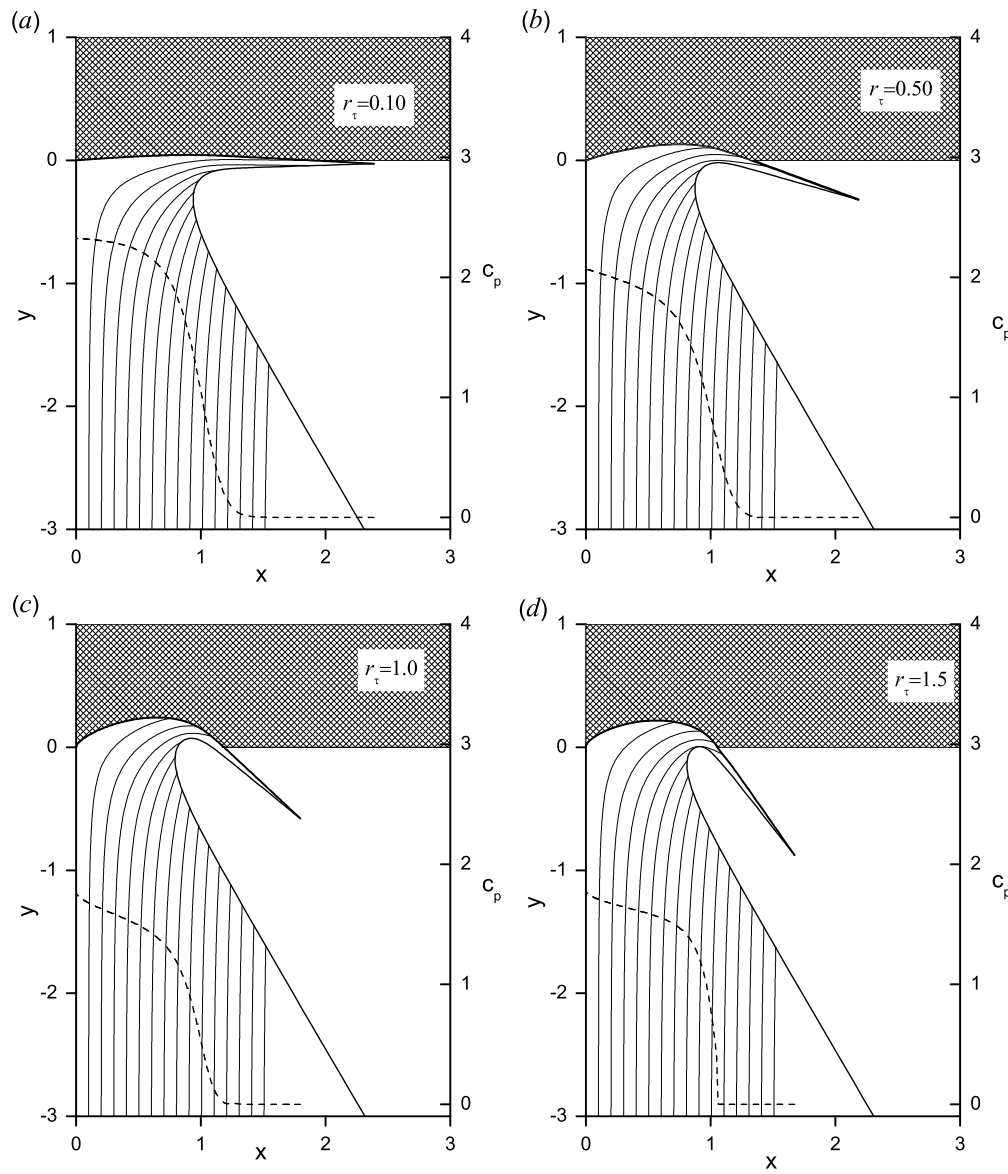

Fig. 5 Impact between a liquid wedge half-angle $\alpha=30^{\circ}$ and an initially flat solid wall $(a)$ $r_{\tau}=0.1,(b) r_{\tau}=0.5,(c) r_{\tau}=1.0,(d) r_{\tau}=1.5$. Streamlines, free surface and the interface (solid lines), and the pressure distribution (dashed lines).

enables the original problem in a physical domain to be reduced to a system of integro-differential equations along the straight lines in the $\zeta$-plane. These equations are solved numerically through the method of successive approximations. The following two case studies have been undertaken: (i) impact between a liquid wedge and an expanding crater in the solid wall; and (ii) impact between a liquid wedge and a cratering wall under the constitute relation of cratering including both the normal and shear stresses acting on the wetted body surface.

The study on liquid impact on a body with the prescribed expanding cavity has revealed two possible flow regimes depending on the rate of the crater expansion. For a liquid wedge of half-angle $\alpha_{A}=10^{\circ}$ and the relative speed of expansion around $0.4<V^{*} / V$, the pressure drops inside the crater from its maximum to zero. This results in a splash jet, which is fully detached from 
the body and is directed almost opposite to the main incoming flow. For ratio $V^{*} / V<0.4$, the upper surface of the splash jet touches the wall and the fully attached model is then assumed. The pressure maxima may then occur outside the crater on the body surface.

Two special cases of cratering have been considered. In the first case the constitutive relation links the rate of cratering and the normal pressure linearly, subject to a prescribed minimal normal velocity of cratering. In this case the shape of the crater is close to a quarter-circle, and in the crater the pressure distribution is approximately constant, and an almost straight line where the prescribed minimal velocity $v_{\min }^{*}$ is applied. In the second case, the constitutive relation links the cratering speed to the shear stress on the wetted body surface linearly. The shape of the crater forms a concave hollow with an acute angle at the stagnation point. In contrast to the first case, this constitute relation leads to a larger speed of cratering near the edge of the crater where the speed of the liquid and the shear stress reach their maximum values.

Acknowledgements This work is supported by Lloyd's Register Foundation (LRF) through the joint centre involving University College London, Shanghai Jiaotong University and Harbin Engineering University, to which the authors are most grateful. LRF supports the advancement of engineering-related education, and funds research and development that enhances safety of life at sea, on land and in the air.

\section{References}

1. Howison SD, Ockendon JR, Wilson SK. (1991) Incompressible water entry problems at small deadrise angles. J. Fluid Mech. 222, 215-230.

2. Howison SD, Ockendon JR., Oliver JM (2002) Deep- and shallow-water slamming at small and zero deadrise angles. J. Engng Maths 42, 373-388.

3. Howison SD, Ockendon JR, Oliver JM (2004) Oblique slamming, planing and skimming. J. Engng Maths 48, 321-337.

4. Korobkin AA (2004) Analytical models of water impact. Eur. J. Appl. Maths 15, 821-838.

5. Oliver JM (2007) Second-order Wagner theory for two-dimensional water-entry problems at small deadrise angles. J. Fluid Mech. 572, 59-85.

6. Dobrovol'skaya ZN (1969) On Some problems of similarity flow of fluid with a free surface. J. Fluid Mech. 36, 805.

7. Zhao R, Faltinsen O (1993) Water-entry of two-dimensional bodies. J. Fluid Mech. 246, $593-612$.

8. Semenov YA and Iafrati A. 2006 On the nonlinear water entry problem of asymmetric wedges. J. Fluid Mech. 547, pp. $231-256$.

9. Wu GX, Sun SL (2014) Similarity solution for oblique water entry of an expanding paraboloid. J. Fluid Mech. 745, $398-408$.

10. Novokshanov R and Ockendon J. 2006. Elastic-plastic modelling of shaped charge jet penetration. Proc. R. Soc. A 462, 3663-3681.

11. Birkhoff G, Macdougall DP, Pugh EM, Taylor GI (1948) Explosives with lined cavities.

J. Appl. Phys. 19, 563-582. (doi:10.1063/1.1698173)

12. Uth T, Deshpande VS (2013) Unsteady penetration of a target by a liquid jet. PNAS 110 (50), 20028-20033.

13. Rehbinder G (1977) Slot Cutting in Rock with a High Speed Water Jet. Int. J. Rock Mech. Min. Sci. \& Geomech. Abstr. 14, 229-234.

14. Semenov YA and Cummings LJ. 2006 Free boundary Darcy flows with surface tension: analytical and numerical study. Euro. J. Appl. Math. 17, 607 -631. 
15. Semenov YA and Wu GX. 2013 Asymmetric impact between liquid and solid wedges. Proc. R. Soc. A 469.

16. Semenov YA, Wu GX, Oliver JM (2013) Splash Jet Caused by Collision of Two Liquid Wedges. J. of Fluid Mech. 737, 132 - 145

17. Newman JN. 1977 Marine hydrodynamics. The MIT press. 418pp. 